\title{
Advanced Direct Digital Synthesis Generator Design for Transuranic Nuclide Alpha Spectrometry Pulses
}

\author{
Guili Peng $\mathbb{C}^{1,2}$ Xianguo Tuo, ${ }^{1,3}$ Huailiang Li, ${ }^{4}$ and Rui Shi ${ }^{3}$ \\ ${ }^{1}$ Fundamental Science on Nuclear Wastes and Environmental Safety Laboratory, \\ Southwest University of Science and Technology, Mianyang 621010, China \\ ${ }^{2}$ School of Control and Mechanical Engineering, Tianjin ChengJian University, Tianjin 300384, China \\ ${ }^{3}$ School of Automation and Information Engineering, Sichuan University of Science \& Engineering, Zigong 643000, China \\ ${ }^{4}$ School of Geophysics, Chengdu University of Technology, Chengdu 610059, China \\ Correspondence should be addressed to Guili Peng; planepeople678@sina.com
}

Received 22 November 2020; Revised 8 January 2021; Accepted 23 January 2021; Published 23 February 2021

Academic Editor: Ghulam Mustafa

Copyright (c) 2021 Guili Peng et al. This is an open access article distributed under the Creative Commons Attribution License, which permits unrestricted use, distribution, and reproduction in any medium, provided the original work is properly cited.

\begin{abstract}
Alpha energy spectrum measurement has been employed in the nuclear waste disposal of transuranic nuclides (such as ${ }^{239} \mathrm{Pu}$ and ${ }^{241} \mathrm{Am}$ ), supervision, and disposal process. The alpha spectrum is made up of alpha particles, which have a fast-moving helium nucleus and an energy of $4-8 \mathrm{MeV}$ with weak penetration ability. Removing alpha particles from radioactive nuclides is an important scientific issue. In this study, a transuranic nuclide alpha particle pulse generator that produces simulated alpha particle pulses similar to real particles was designed. Field programmable gate array (FPGA) was adopted as its core chip and we obtained the digital pulse waveform using software tracing points while simulating real alpha particles by random numbers. Accordingly, the alpha energy spectrum of a radioactive source ${ }^{241} \mathrm{Am}$ was obtained using a passivated ion-implanted planar silicon (PIPS) detector. Afterward, the alpha particle was extracted from the energy spectrum and was then compared to the alpha particle pulse of the two methods, deriving a result. Here, both groupings of particle pulse waveforms were found to be very similar, and the periodic error of the particle was observed to be less than $1 \%$. Furthermore, the amplitude and time interval of the particle were apparently similar to the actual spectrometry pulse.
\end{abstract}

\section{Introduction}

Numerous transuranic nuclides exist in nuclear facility operations, accidents, decommissioning, decontamination, storage of nuclear waste, and the disposal process of recycling. In these projects, radioactive particles may be produced, forming radioactive aerosols in the air, which include alpha, beta, and gamma radioactive aerosols [1]. The alpha particles dispersed hold a particularly important position amongst the three particles. Accurate alpha particle pulse data accuracy is imperative, and a method for the effective measurement of alpha particles is necessary. Alpha particle pulses can provide a scientific basis for the implementation of nuclear facility decommissioning and nuclear waste disposal $[2,3]$. The technology of alpha particle measurements, analysis, and identification has rapidly developed over the last ten years [4-7]. Alpha-ray measurement is an important method used for the analysis of alpha particles $[8,9]$. For a long time, researchers surveyed alpha particle pulses [10]; however, the penetration of alpha particles is very weak and gets absorbed by the medium before reaching the detector $[11,12]$. Alpha particle measurement technology is very difficult to master [13]. At present, passivated implanted planar silicon (PIPS) detectors have been widely used to perform alpha particle measurement. Thus, alpha particle pulses may be analyzed, and alpha particle pulse characteristics may be observed. Currently, the study of alpha particle waveforms is still in its infancy and cannot be detected accurately. Research has demonstrated that the decay of the radioactive nucleus is random and occurs over time. Moreover, nuclear decay obeys the Poisson distribution $(\mathrm{Pd})$ in average unit time, in which charged particles (i.e., alpha and Beta) follow this rule. Alpha particles are emitted during radiating alpha decay in an unstable nucleus, 
which also takes place randomly over time [14]. Hence, the alpha particle pulse belongs within Poisson distribution.

This paper puts forward a digital pulse generator focused on alpha particles. The generator simulates the statistical properties of the alpha particle pulse and obtains a standard alpha particle waveform [15]. Although the alpha particle numbers are random, amongst equal time interval distributions, the probability of generating pulses is equal at an arbitrary time interval on the timeline. They are also different in amplitude; however, the waveform amplitude is related to the energy of the alpha particle [16]. This study considers the characteristics of alpha particle waveforms, the time interval random sequence, and the standard of the amplitude characteristics of disparate energy alpha particles. Simultaneously, the generator is able to simulate the entire alpha particle pulse. To this effect, we have attempted to obtain the true alpha particle pulse using a passivated implanted planar silicon (PIPS) detector in a laboratory setting. Finally, the two pulses were compared, the errors were calculated, and the characteristics of alpha particle pulses were analyzed.

This study proposes a simulated algorithm for the nuclear pulse of alpha particles. Accordingly, it establishes a mathematical function in order to simulate the pulse according to the real alpha particle pulse's characteristics' parameters. This function was programmed using MATLAB in order to draw pulse waveforms and generate pulse data. Researchers can change the parameters of the function to alter the pulse waveforms. Finally, the data were put from the MATLAB program into the ROM of field programmable gate array (FPGA), after which direct digital synthesis (DDS) technology was used to simulate alpha pulse waveforms. This study innovatively utilizes mathematical functions so as to simulate the nuclear signal rather than using the tracing point diagram method. Hence, this technique is convenient in altering the waveforms, enabling it to more truly reflect the characteristics of the nuclear pulse.

\section{Method}

2.1. Alpha Particle Pulse Generator. Alpha particles have a fast-moving helium nucleus with an energy of $4-8 \mathrm{MeV}$, which is emitted from transuranic nuclides such as ${ }^{239} \mathrm{Pu}$ and ${ }^{241} \mathrm{Am}$. When an alpha particle passed through the detector, the detector material absorbed its energy. Following absorption, the alpha particle electrical pulse signal was obtained. By analyzing the captured electron pulses, the alpha particle's characteristics were subsequently acquired. The alpha particle pulse period was from $35 \mu \mathrm{s}$ to $40 \mu \mathrm{s}$, and the pulse width range was from $10 \mu$ s to 15 [17]. Moreover, the rise time range was from $3 \mu$ sto $5 \mu$ s, while the down time range was from twenty-three to twenty-eight microseconds.
Its time interval and pulse amplitude were random, and the maximum amplitude of the pulse was about 100 millivolts.

Tracing point graph is a new method of drawing image, which aims to realize MIF file. The MIF file can be used to store simulated alpha pulse signal image and can be directly connected with ROM of the FPGA. We convert MIF image into data and put into ROM. The image of tracking point graph method needs to follow the characteristics of alpha pulse signals and is drawn in MIF_Make 2010 software.

Direct digital synthesis technology (DDS) is that which converts a series of digital signals into analog signals through the D/A converter. This technology mainly uses ROM look-up table method. It only needs to store the amplitude sequence corresponding to different phases in ROM and then address it through the output of phase accumulator. After digital to analog conversion and low-pass filtering (LPF) output, the desired analog signal can be obtained. DDS is mainly composed of standard reference frequency source, phase accumulator, waveform memory, and D/A converter.

\subsection{Time Interval Random Algorithm Implementation.} The alpha particle numbers were random. When the pulses were generated with a constant speed, the time intervals between the two adjacent pulses were different. This time interval was regarded as a random variable, which obeyed a certain distribution. A pseudorandom number was then generated in order to replace the time interval using a computer program. The common algorithm adopted the linear congruence (LC) method, established in 1951 by Lehmer, which generated uniformly distributed random numbers [18].

The formula is as follows:

$$
\begin{aligned}
X_{n} & =\left(a x_{n-1}+c\right) \bmod M, \\
r_{n} & =x_{n} \bmod M .
\end{aligned}
$$

The algorithm was made up of four parameters: modulus $M(M>0)$, multiplier $a(0 \leq a<\mathbf{M})$, incremental $c(0 \leq c<M)$, and initial value (Seed) $X_{0}\left(0 \leq X_{0}<M\right)$, obtaining a random sequence $\left\{X_{n}\right\}$ via iterative formula (1), where $0 \leq X_{n}<M$. If the condition satisfies $0 \leq X_{n}, X_{n+1}<M$, the generating sequence must be periodic. The parameters $a, c$, and $M$ are key to produce $a$ more accurate random number. The parameter $c$ in a random sequence has no effect on the pseudorandom number; hence, $c=0$ simplifies the algorithm. To avoid algorithm overflow, the algorithm must be improved.

The formula is as follows:

$$
f(X)=(a \cdot X) \bmod M=P_{1}(X)+M P_{2}(X),
$$

where in

$$
\begin{aligned}
& P_{1}(X)=a \cdot\left(X \bmod \left[\frac{M}{a}\right]\right)-(M \bmod a) \cdot\left[\frac{X \cdot a}{M}\right], \quad\left|P_{1}(X)\right| \leq M-1, \\
& P_{2}(X)=\left[\frac{X \cdot a}{M}\right]-\left[\frac{a \cdot X}{M}\right], \quad P_{2}(X)=0 \text { or } 1 .
\end{aligned}
$$


Then,

$$
\begin{aligned}
& f(X)=P_{1}(X), \text { when } P_{2}(X)=0, \quad 1 \leq P_{1}(X) \leq M-1, \\
& f(X)=P_{1}(X)+M, \text { when } P_{2}(X)=1, \quad-M+1 \leq P_{1}(X) \leq-1 .
\end{aligned}
$$

These findings demonstrated advanced linear congruence algorithms, including mixed linear congruence (MLC) and prime modulo multiplicative linear congruence (PMMLC).

When $C>0$ in formula (1), it is called MLC.

Chosen algorithm parameters are as follows:

$$
\left\{\begin{array}{l}
M=2^{L}, a=4 \alpha+1, \\
c=2 \beta+1, \\
x_{0},
\end{array}\right.
$$

where the parameters $\alpha, \beta$ are nonnegative integers.

The MLC recursive formula is as follows:

$$
\left\{\begin{array}{l}
x_{n}=\left((4 \alpha+1) x_{n}-1+(2 \beta+1)\right)\left(\bmod 2^{L}\right), \\
r_{n}=\frac{x_{n}}{2^{L}}, \quad(n=1,2, \ldots \ldots) .
\end{array}\right.
$$

When the parameters $a$ and $M$ are positive integers and coprime, it is called PMMLC. The minimum $v$ is the order of $M$ in the formula $a^{v} \equiv 1 \bmod M$. When $v$ is satisfied, $v=M-1$, so $a$ is the prime element of $M$. Using the principle of spill over to realize the PMMLC algorithm:

The parameter $M=2^{L}-g$ is the maximum prime in less than $2^{L}$; the formula is as follows:

$$
x_{n}=a x_{n}-1\left(\bmod 2^{L}-g\right) \text {, }
$$

where we order the parameters as $z_{n}=a x_{n}-1\left(\bmod 2^{L}-g\right)$, $k=\left(a x_{n}-1 / 2^{L}\right)$, and derive the advanced recursive formula as follows:

$$
x_{n}= \begin{cases}z_{n}+\mathrm{kg}, & z_{n}+\mathrm{kg}<2^{L}-g, \\ z_{n}+\mathrm{kg}-\left(2^{L}-g\right), & z_{n}+\mathrm{kg} \geq 2^{L}-g .\end{cases}
$$

2.3. Amplitude Random Implementation. Alpha particles are not only random in time intervals, but they are also random in amplitude $[19,20]$. After an alpha particle is converted to an electronic signal from the detector, the statistical fluctuations of the electronic pulse amplitude become random. Therefore, alpha particle pulse amplitudes obey Gaussian distribution.

The mathematical equation is as follows:

$$
P(v)=\frac{1}{\sqrt{2 \pi \delta}} e^{-(v-\bar{v})^{2} / 2 \delta^{2}}
$$

The generator produced a large classic Gaussian pseudorandom number using the polar algorithm [21]. The Gaussian number replaced the pulse amplitude of the alpha particle, and its main principle may be outlined as follows. Independent random variables were adopted in $\left(X_{1} \in N(0,1), X_{2} \in N(0,1), \quad X_{1}^{2}+X_{2}^{2} \in \chi^{2}(2)\right)$ order to calculate the density function.

$$
f(X)=a e^{-(x / 2)} \text {, in it } X=X_{1}^{2}+X_{2}^{2} .
$$

After the density function an independent Gaussian random number was produced,

$$
\begin{aligned}
& D=X^{2}+Y^{2}, \\
& X \in U(0,1), \\
& Y \in U(0,1) \\
& f=\sqrt{-2 \frac{(\log D)}{D} .}
\end{aligned}
$$

Then, the results $(f \times X, f \times Y)$ from formula (9) were utilized, which are a pair of independent Gaussian random numbers.

A uniform random number was then obtained by obeying Gaussian distribution, which transformed the method derivation process when acquiring two uniform random variables $U_{1}$ and $U_{2}$ within the interval $(0,1)$. The transformed function is given as follows:

$$
\begin{aligned}
& Y=R \cos \theta=\sqrt{-2 \ln U_{i}} \cos \left(2 \pi U_{2}\right), \\
& Y=R \sin \theta=\sqrt{-2 \ln U_{i}} \sin \left(2 \pi U_{2}\right) .
\end{aligned}
$$

The function in the Box-Muller derivation process is formulated as follows.

$$
\begin{aligned}
R^{2} & =x^{2}+y^{2}, \\
\theta & =\arctan \left(\frac{x}{y}\right) .
\end{aligned}
$$

Then, the parameters of the Gaussian random number are obtained as follows:

$$
\begin{aligned}
e & =-2 \ln \left(U_{1}\right), \\
R & =\sqrt{e}, \\
g_{1} & =\sin \left(2 \pi U_{2}\right), \\
g_{2} & =\cos \left(2 \pi U_{2}\right), \\
x_{1} & =f * g_{1}, \\
x_{2} & =f * g_{2} .
\end{aligned}
$$


2.4. Generator Hardware Architecture. The alpha particle pulse generator adopted the field programmable gate array (FPGA) as the core chip. The FPGA chip has advantages of low cost, high density, short design cycle, and repeatable programming [22]. Moreover, it has a million logic gate resources and powerful hardware computing power. The processing speed and FPGA fit the requirements of the alpha particle electronic pulse $[23,24]$.

In this approach, the cyclone family EP2C8Q208C8N FPGA of Altera Company was packaged in PQ208 with a capacity of approximately 150000 gates. This FPGA configuration contained PROM chip EPCS1S and EPCS4S, and an external $+5 \mathrm{~V}$ power source was supplied to the FPGA core board. Using the LM 317 chip, the $+5 \mathrm{~V}$ power was turned into $+3.3 \mathrm{~V}$ and $+1.5 \mathrm{~V}$ so as to supply the FPGA chip. The FPGA core board has a $50 \mathrm{MHz}$ crystal oscillator to supply standard clock signals as well as $138 \mathrm{I} / \mathrm{O}$ ports and two programming interfaces to download the FPGA and PROM. These configurations are convenient in selection for different users.

Next, conversion of the alpha particle digital pulses into analog electronic pulses was necessary. Therefore, the alpha particle pulse generator utilized a high-speed digital-toanalog converter (DAC), where the DAC900 E DAC chip of Texas Instruments ${ }^{\circledR}$ (TI) was used, which has a high-performance conversion rate of 10 -bit resolution. The DAC chip is available in a TSSOP-28 package with an update rate greater than 165 MSPS. MSPS means million samples per second. It is the unit of $\mathrm{A} / D$ conversion rate. This DAC $900 \mathrm{E}$ DAC board adopted a single-ended DC output power method, which provided positive and negative bipolar output power and was connected to a OPA690 chip operational amplifier at the end. Modifications of the ratio resistance were made in order to adjust the output voltage amplitude, ranging from $-5 \mathrm{~V}$ to $+5 \mathrm{~V}$, as an option.

\section{Experiment and Simulation Result}

3.1. Experiment Result. The testing of the alpha particle pulse was carried out using an alpha spectrometer at the Fundamental Science on Nuclear Wastes and Environmental Safety Laboratory at Southwest University of Science and Technology. The spectrometer was produced by ORTEC ${ }^{\circledR}$ (USA), utilizing the passivated implanted planar silicon (PIPS) detector for testing [25]. This detector adopted threemicron complementary metal-oxide-semiconductor transistor (CMOS) technology and can accurately determinate the geometric size of the detector while controlling oxide passivation and ion implantation [26-28].

The PIPS detector and alpha spectrometer are shown in Figure 1.

The principle utilized the PIPS detector $[29,30]$, which is the complex interaction between incident particles and the depletion layer crystal detector. The detector produced the junction capacitance C. For the silicon detector, the junction capacitance $\mathrm{C}$ function is as follows:

$$
C=\frac{2.1 S}{\sqrt{\rho V}}\left(\frac{\mathrm{pf}}{m m^{2}}\right)
$$

When the surface area of the alpha detector was about $0.06 \mathrm{~cm}^{2}$, the resistivity $(\rho)$ was $1000 \Omega \cdot \mathrm{cm}$ and the offset voltage was 50 volts. According to formula (15), the capacitance was calculated to be 5.6PF. For an alpha particle of about $5.48(56 \mathrm{MeV})$, an ionization energy of about $3.62 \mathrm{eV}$ was observed when measured in the PIPS detector.

The formula for calculating the electrostatic charge is given as follows:

$$
Q=\frac{5.4856 \times 10^{6} \times 1.6 \times 10^{-19}}{3.62}=2.4246 \times 10^{-13} \mathrm{C} \text {. }
$$

The formula for calculated amplitude of the alpha pulse from this detector is as follows:

$$
V=\frac{Q}{C}=\frac{2.4246 \times 10^{-13}}{5.6 \times 0^{-12}}=0.0433 \mathrm{~V}=43.3 \mathrm{mV} \text {. }
$$

According to the abovementioned experimental conditions, a radioactive source of ${ }^{241} \mathrm{Am}$ was adopted for measurement in the laboratory. For the alpha spectrometer, vacuum degree and distance were considered. When the vacuum degree was 300 Torr and the detection range was $24 \mathrm{~mm}$, an alpha particle pulse of ${ }^{241} \mathrm{Am}$ was measured by the detector. After passing the filter, linear amplifier, analog to digital converter (ADC), the waveform of the pulse was recorded in the oscilloscope. The pulse waveform from the PIPS detector is shown in Figure 2.

3.2. Simulation Result in MATLAB. After analyzing the shape of the alpha pulse, the standard alpha particle waveforms were assumed to be approximated coincidence functions of the Gaussian and antiexponential functions. It has Gaussian distribution properties and exponential tailing, and the main pulse forming was an exponential decay signal, which may be described by a piecewise function [31-33].

The function is as follows:

$$
\left\{\begin{array}{l}
f(x)=\frac{1}{\sqrt{2 \pi \sigma}} \exp \left(-\frac{(x-\mu)^{2}}{2 \sigma^{2}}\right), \quad 0<x<t_{1}, \\
f(x)=a \cdot b^{(x-c)}, \quad 0<b<1, c>0 . t_{1}<x<t_{2} .
\end{array}\right.
$$

When detecting the ${ }^{241} \mathrm{Am}$ alpha particle data form experiment using the Si semiconductor detector, the undetermined coefficient method was utilized in order to find out the parameters of the equation. The "undetermined coefficient method" means a method to solve the unknown parameters of equation (18). Some unknown coefficients in formula 18 are set, and these coefficients are determined using the coefficients of the similar terms which are equal in two polynomial identities, so as to obtain the coefficients. The parameters were found to be as follows:

The parameters are that $\mu=10, \sigma=9.947 \times 10^{-5}$, $a=2.5, c=50$.

The alpha pulse's cycle is about $30-50 \mu s$, and the half width is about $10 \mu \mathrm{s}$. So, we choose the $t_{1}=10 \mu \mathrm{s}$ and $t_{2}=50 \mu \mathrm{s}$ in equation (19). Put the parameters into formula (17), and get the function as follows: 

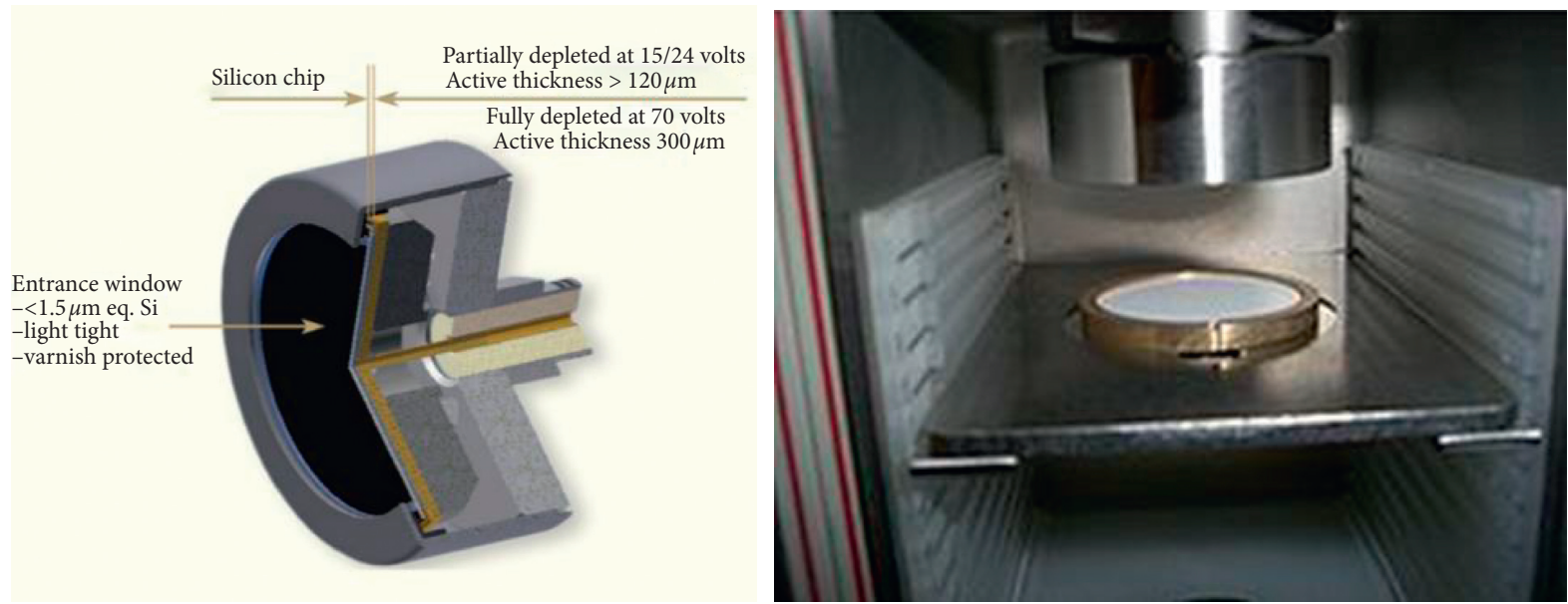

FIgURE 1: PIPS detector and ORTEC $^{\circledast}$ alpha spectrometer.

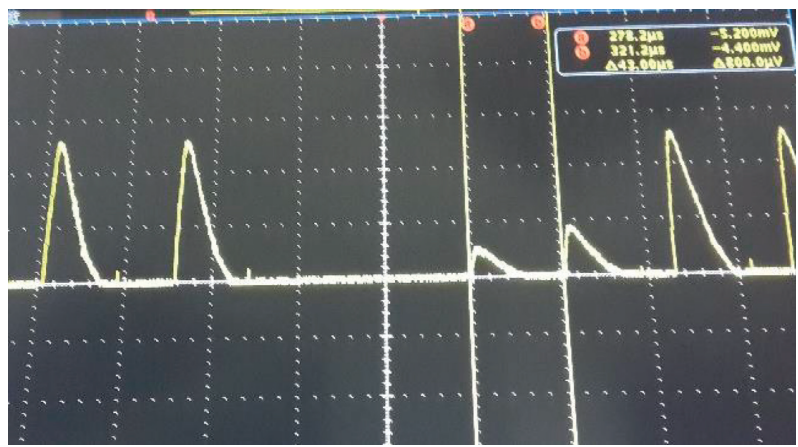

Figure 2: Alpha pulse waveform from the PIPS detector.

$$
\left\{\begin{array}{l}
f(x)=-\frac{1}{\sqrt{2 \times 9.947 \times 10^{-5} \pi}} \exp \left(-\frac{(x-10)^{2}}{2 \times 9.947 \times 10^{-5^{2}}}\right), \quad 0<x<10, \\
f(x)=\frac{5}{2} \cdot 0.933^{(x-50)}, \quad 10<x<50 .
\end{array}\right.
$$

In this study, the alpha particle pulse use formula (17) was realized as a single waveform, and the program was then compiled in MATLAB. Figure 3 shows the standard pulse with white Gaussian noise and low frequency noise. The low frequency noise in nuclear pulse mainly refers to the noise that increases with the decrease of efficiency. It is usually the gate noise, transistor noise, field effect transistor noise, and resistance noise. It mainly depends on the surface condition of the device, mostly low frequency flicker. Its frequency is mostly $10-100 \mathrm{~Hz}$.

\section{Generator Test Results}

The alpha particle pulse generator was divided into three steps. First, a single pulse was obtained. Second, multiple pulses at a random amplitude were acquired. Finally, a complete pulse waveform with an amplitude and random interval was obtained. The circuit was designed in the FPGA chip in order to gradually implement the functions of the three steps.
We have designed two random number generation modules called ROM "aaa" and "ccc." Gaussian random number is used to simulate the random amplitude of alpha pulse and stored in ROM "aaa." The linear congruence random number is used to simulate the time interval of alpha pulse and stored in ROM "bbb."

The circuit in FPGA is shown in Figure 4.

Step 1. Single pulse test results.

A single alpha particle pulse was acquired using MATLAB and a dataset on the software was mapped out with a length of 1024 and a width of 8 . The dataset length is 1024 which means the image has 1024 sample points in horizontal ordinate. The width is 8 which means the image has 8 sample points in vertical coordinates. The maximum amplitude is $0 \times 100$ in hexadecimal. The sampling frequency was $1 \mathrm{kHz}$, and the amplitude was $43.2 \mathrm{mV}$, purposely not exceeding $100 \mathrm{mV}$, so the digital amplitude data were not more than $0 \times 80$ hexadecimal. 


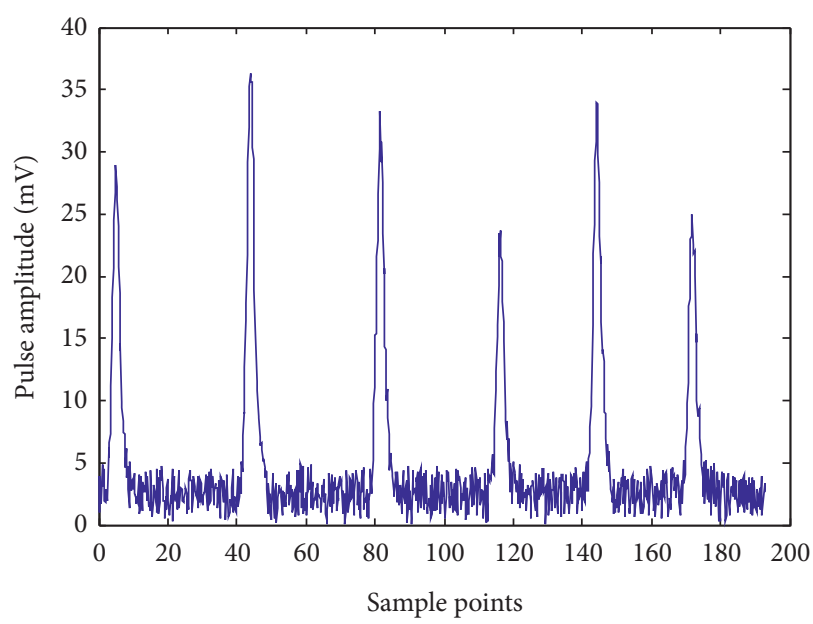

(a)

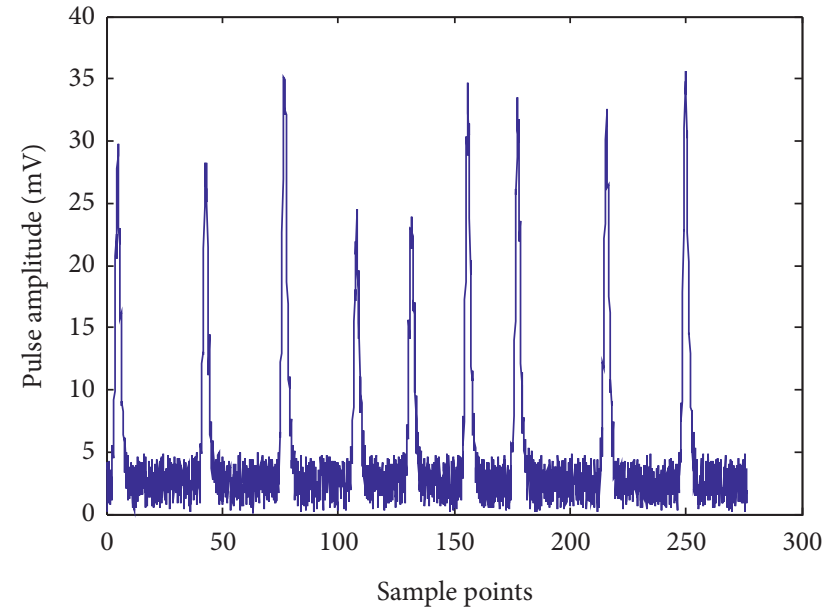

(b)

Figure 3: (a) Alpha pulse adds white Gaussian noise. (b) Alpha pulse adds low frequency noise.

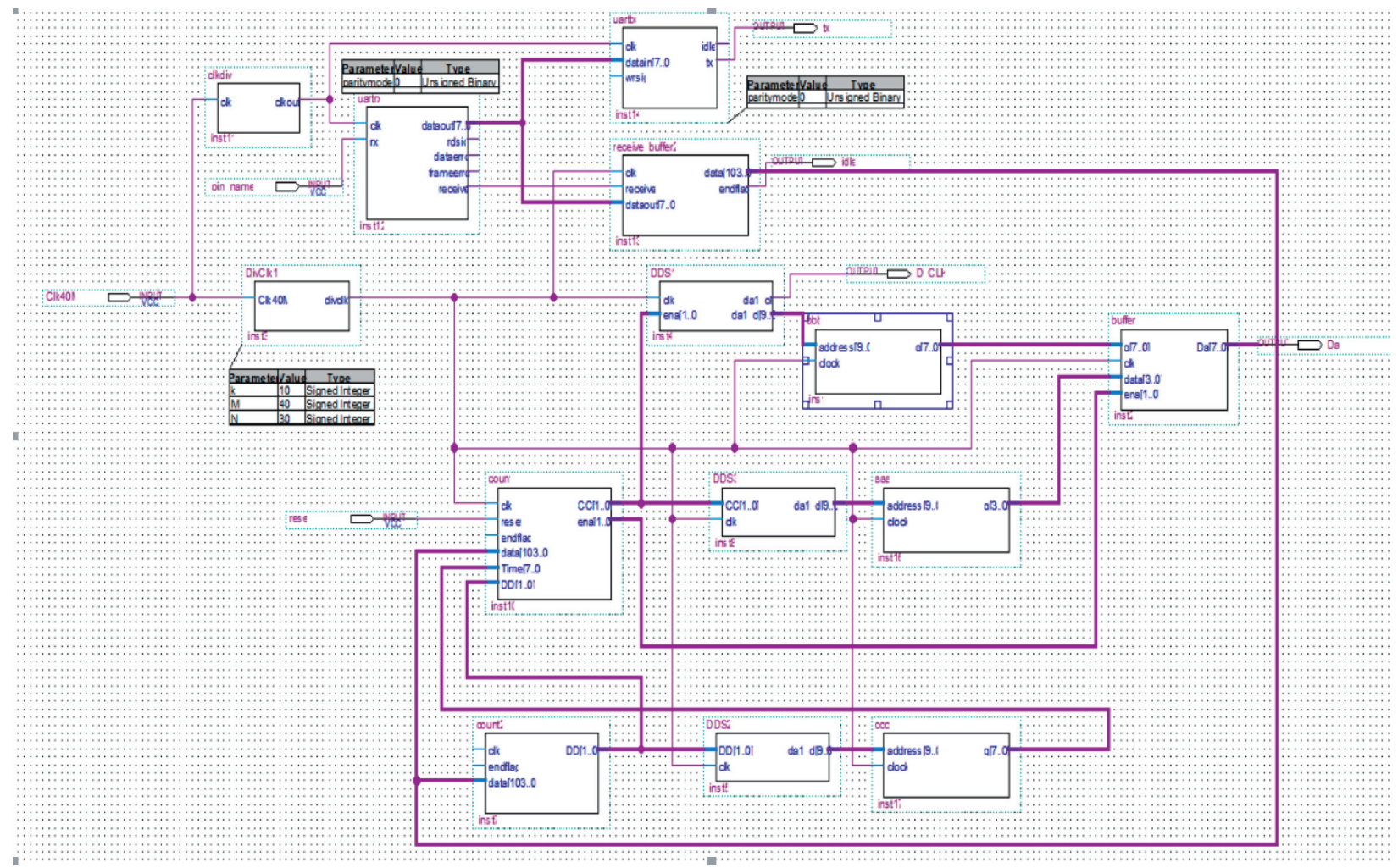

FIGURE 4: Alpha particle pulse generator circuit in FPGA.

The generated pulse data were then input to the FPGA ROM. According to the captured time of the alpha particle pulse, the average pulse was designed by the direct digital synthesizer (DDS) program in FPGA, in which the pulse average period was $35 \mu$ s. Through the calculation, the counter variable CNT was set to 1024 points so that the corresponding time was $35 \mu \mathrm{s}$ in program. The counter variable CNT was 2249 points so that the corresponding time was $75 \mu \mathrm{s}$, which concludes the complete pulse with the time interval. The purpose was to obtain a single alpha pulse waveform without overlapping it. Figure 5 shows a single pulse test result.

The alpha particle pulse was displayed in an oscilloscope, and it increased rapidly but declined slowly and had a trailing. Its cycle was $34.88 \mu \mathrm{swith}$ time interval of $40 \mu \mathrm{s}$. The alpha particle pulse amplitude value was $54.8 \mathrm{mV}$. All in all, the shape and characteristic data of the pulse were found to be consistent with our design. 


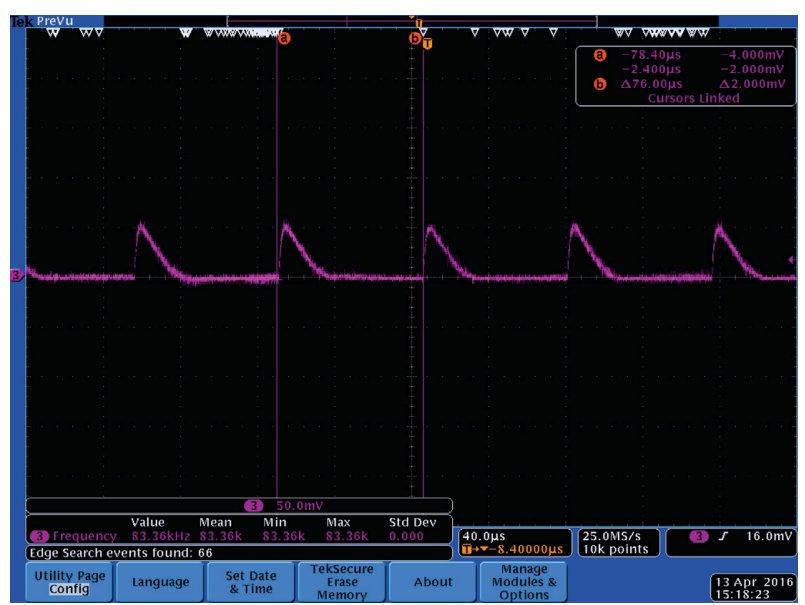

Figure 5: One single alpha particle pulse test result.

Step 2. Multiple pulse test results at random amplitude.

In order to realize multiple pulses with a random pulse amplitude, the polar algorithm was used to generate Gauss random numbers. The generated random numbers were encapsulated in a "Gauss" program module in FPGA. Figure 6 shows the multiple pulse with random amplitude.

The alpha particle pulse was displayed through the oscilloscope. While the amplitude of the pulse waveform was from $20 \mathrm{mV}$ to $55 \mathrm{mV}$ and the shapes did not overlap, the shape and characteristic data of the pulse were found to be consistent with our design.

Step 3. Multiple pulse test results at random amplitude and random time interval.

To realize the complete pulse, time interval random numbers were established using the linear congruence (LC) algorithm. The random numbers were encapsulated in a "LCG" program module in FPGA. The counter variable CNT was then set to 1024 with a corresponding time of $35 \mu \mathrm{s}$. Moreover, in order to ensure that the pulse interval was greater than $35 \mu \mathrm{s}$, the counter variable CNT was set in the "LCG" program module between 1024 and 4096. When the counter variable CNT was set in this manner, the cycle of random numbers was ensured to be greater than $35 \mu \mathrm{s}$. The complete alpha particle pulse is shown in Figure 7.

The alpha particle pulse was completely nonoverlapping, and its minimum time interval was $10 \mu \mathrm{s}$ with a maximum time interval of $80 \mu s$. The half width of the alpha particle pulse was equal, while the amplitude of the pulse waveform was from $20 \mathrm{mV}$ to $55 \mathrm{mV}$. The shape and characteristic data of the pulse were found to be consistent with our design.

\section{Discussion}

This study attempted to simulate the transuranic nuclide alpha particles pulse. Here, the alpha particle pulse generator was designed in three steps: a single pulse, multiple pulse with random amplitude, and multiple pulse with random amplitude and random time interval. The single pulse data were obtained using MATLAB, and the circuit was designed by FPGA. The Gauss random number was obtained using
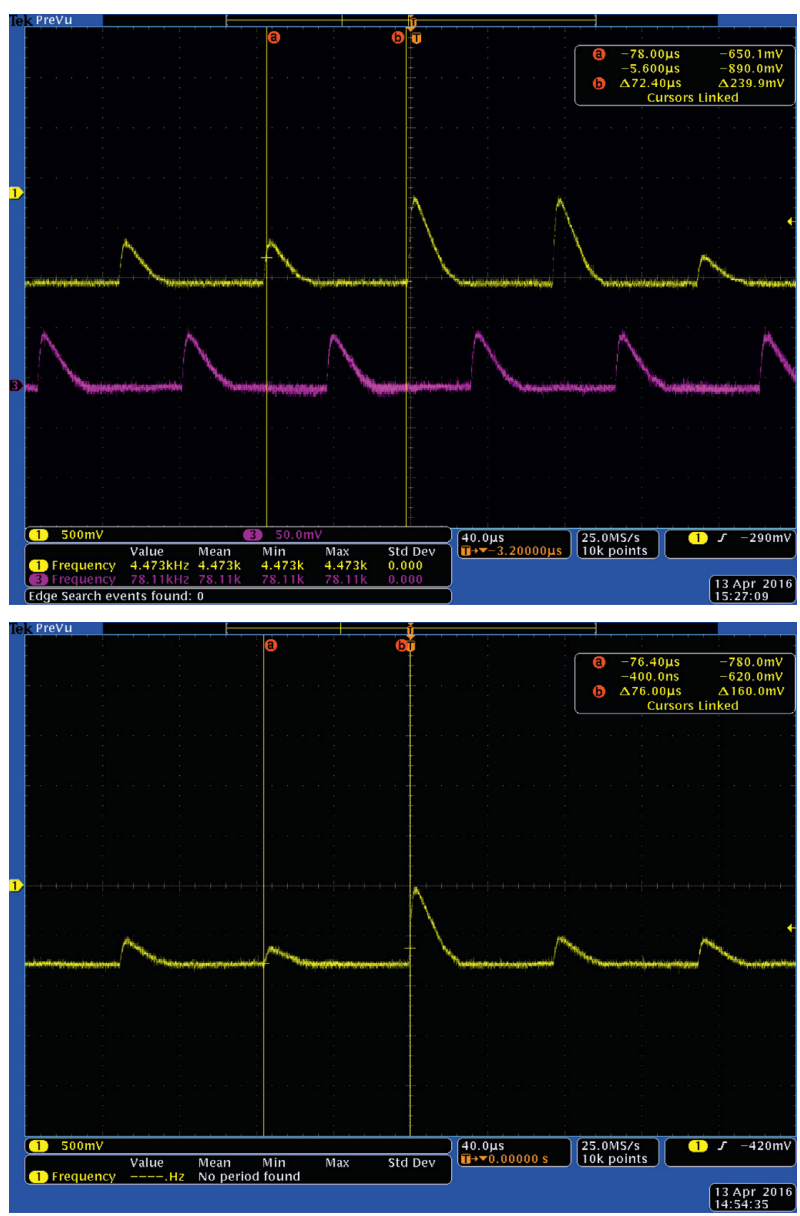

FIgURE 6: Multiple pulse waveform at random amplitude.

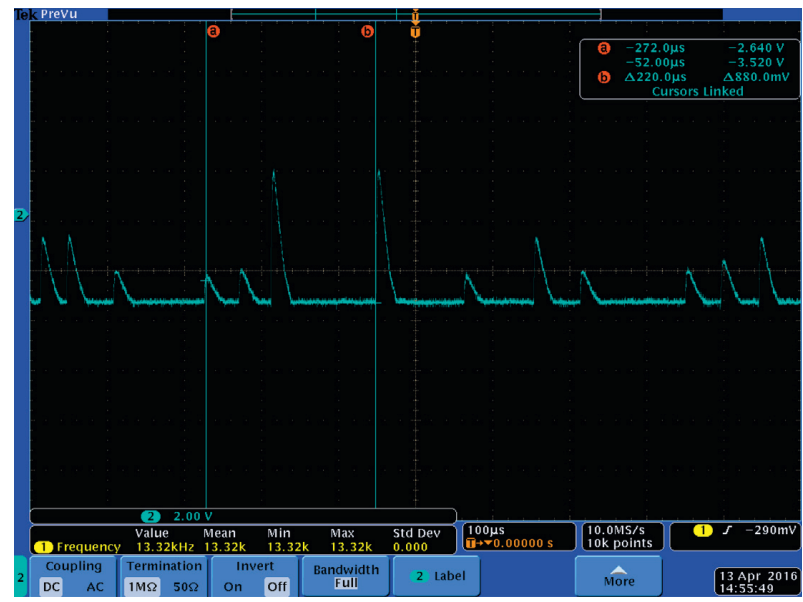

FIgURE 7: The complete alpha particle pulse waveform.

the polar algorithm, where multiple pulses with arbitrary amplitude were attained. Finally, an alpha particle pulse using the linear congruence (LC) algorithm was used to obtain the random number of the time interval. Figure 8 shows three alpha pulse waveforms on one screen.

Based on the pulse generator of the super uranium radionuclide alpha particle, the pulse shape of alpha particle 


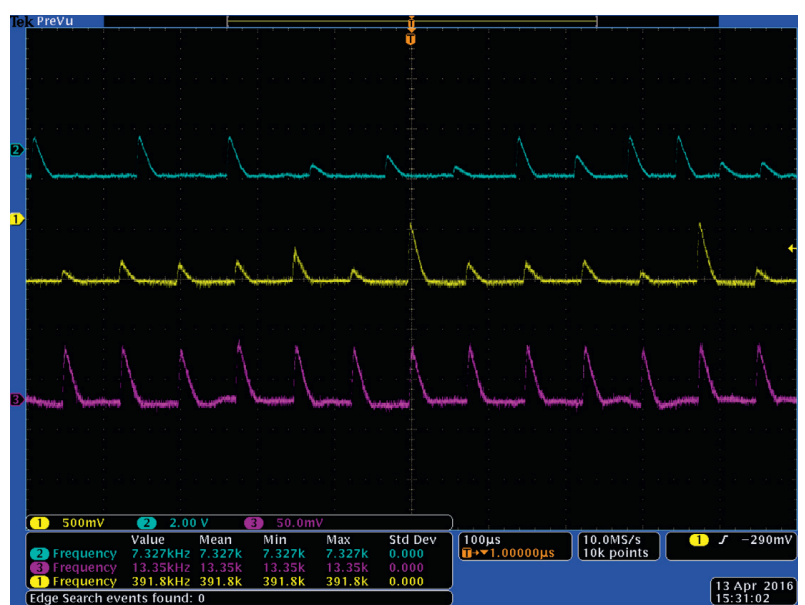

FIGURE 8: Generated alpha particle pulse waveform.

TABLE 1: Comparison of alpha particle pulse signal parameters.

\begin{tabular}{lccc}
\hline & $\begin{array}{c}\text { Pulsevalue } \\
\text { in lab }\end{array}$ & $\begin{array}{c}\text { Generated } \\
\text { pulse }\end{array}$ & $\begin{array}{c}\text { Relative } \\
\text { error }\end{array}$ \\
\hline Cycle (us) & 35.25 & 34.88 & 0.0104 \\
Rise time (us) & 3.22 & 4.01 & 0.2452 \\
Fall time (us) & 30.44 & 29.76 & 0.0223 \\
Pulse width (us) & 10.24 & 9.66 & 0.0566 \\
Random amplitude (mV) & 43.3 & 54.8 & 0.2425 \\
Max amplitude (mV) & 48 & 53.5 & 0.1146 \\
Min amplitude (mV) & 21 & 25.3 & 0.2047 \\
\hline
\end{tabular}

was concluded to grow rapidly, slowly decreasing with a large tail. Accordingly, it had a period of 34.88 us and a maximum pulse amplitude that did not exceed $100 \mathrm{mV}$. In addition, the alpha particle pulse possessed a certain time interval, and the half width was equal.

The new issue, as discussed, concerns the relationship between the alpha particle pulse data, which used the PIPS detector in the laboratory, and the instrument generated. According to the obtained data regarding alpha particle pulses in laboratory, such as pulse cycle, rise time, fall time, half width, time interval, and amplitude, the alpha particle pulse program was rebuilt in FPGA. The experimental results demonstrated that the alpha particles pulse waveform on the oscilloscope had similarity and consistency. However, due to the randomness and uncertainty of the real alpha particle pulse, the PIPS detector found it difficult to capture the full alpha particle pulse signal. Hence, the more representative and complete pulse signal was chosen in the oscilloscope, as shown in Table 1.

When comparing the two alpha particles pulses, the error of the single pulse time parameter was quite scant, as noted. The instrument generated pulse signal was able to approximate the alpha particle in cycle, fall time, and half width. The difference in particle energy led to a different amplitude of data. Since only one pulse amplitude was selected, it was unable to replace all alpha particle pulse signals, representing a sizable error. Accordingly, further experimentation is needed in the future in order to ascertain the amplitude rules. Furthermore, more accurate information regarding the amplitude of the alpha particle pulse signal should be obtained.

\section{Conclusion}

A preliminary study regarding the alpha particle generator was presented in this study. Here, the circuit program was designed in FPGA, and Gauss random numbers were calculated using the polar algorithm to obtain the alpha particle pulse amplitude, after which the uniform distributed random numbers were generated using the LC algorithm. The pulse time interval was then obtained, and the alpha pulse data were provided using the PIPS detector in the laboratory and simulated using MATLAB. Moreover, the alpha particle pulse data were imported into the FPGA ROM to complete the generator's design. When comparing the three kinds of pulse data, it was concluded that the error of pulse data was insignificant.

Currently, the as designed alpha spectrometry pulse generator is not perfect, and some defects exist. Essentially, a noise signal is present on the alpha pulse waveform with a bit of burr in the pulse shape. Additionally, errors were noted in the alpha particle pulse data, in which some parameter errors were greater than ten percent. However, this study may improve circuit design in the future to enhance the accuracy of alpha pulse parameters and reduce any corresponding errors.

\section{Data Availability}

All data, models, and code generated or used during the study are available in the submitted article. Some codes generated or used during the study are available from the corresponding author upon request. The data are generated by MATLAB function, and we can change through altering the characters.

\section{Conflicts of Interest}

The authors confirm that there are no conflicts of interest regarding the publication of this paper.

\section{Acknowledgments}

This work was supported by the National Natural Science Foundation of China (nos. 42074218 and 41874213) and Fundamental Science on Nuclear Wastes and Environmental Safety Laboratory Open Foundation (no. 17kfhk04).

\section{References}

[1] A. Noureddine, B. Baggoura, J. J. Larosa, and N. Vajda, "Gamma and alpha emitting radionuclides in some Algerian soil samples," Applied Radiation and Isotopes, vol. 48, no. 8, pp. 1145-1148, 1997.

[2] A. Baeza, J. Guillén, and J. W. Mietelski, "Uptake of alpha and beta emitters by mushrooms collected and cultured in Spain," Journal of Radio analytical and Nuclear Chemistry, vol. 261, pp. 375-380, 2004.

[3] A. Baeza, J. A. Corbacho, R. Pérez et al., "A new approach to the analysis of alpha spectra based on neural network 
techniques," Nuclear Instruments and Methods in Physics Research Section A: Accelerators, Spectrometers, Detectors and Associated Equipment, vol. 652, no. 1, pp. 450-453, 2011.

[4] Z. Li, T. Xianguo, and S. Rui, "Analytic fitting and simulation methods for characteristic X-ray peaks from Si-PIN detector," Nuclear Science and Techniques, vol. 24, no. 6, Article ID 60206, 2013.

[5] T. Xianguo, Z. Li, and K. Mu, "Alpha contamination monitoring instrument based on LRAD technique for complex surface monitoring," Journal of Nuclear Science and Technology, vol. 45, pp. 282-285, 2008.

[6] W. B. Divon and B. Rozen, "A random pulse generator," Nuclear Instruments and Methods, vol. 39, no. 1, pp. 77-87, 1966.

[7] Y. I. Cheng, T. Xianguo, Z. Li et al., "Measuring energy loss of alpha particles in different vacuum, conditions," Nuclear Science and Technique, vol. 22, pp. 146-150, 2011.

[8] A. Martín Sánchez and F. Vera Tomé, "An experimental study of symmetric and asymmetric peak-fitting parameters for alphaparticle spectrometry fitting parameters for alpha-particle spectrometry," Nuclear Instruments and Methods in Physics Research Section A: Accelerators, Spectrometers, Detectors and Associated Equipment, vol. 339, no. 1-2, pp. 127-130, 1994.

[9] A. Bland, F. Vera Tomé, and P. Rubio, "Fitting of alpha spectra. Application to low-level measurements," Applied Radiation and Isotopes, vol. 47, no. 9-10, pp. 899-903, 1996.

[10] J. Rubio Montero, A. Pertti, and T. Jorma, "SAMPO80: An accurate gamma spectrum analysis method for minicomputers," Nuclear Instruments and Methods, vol. 190, no. 1, pp. 89-99, 1981.

[11] E. Garciatorano, "A model shape for the analysis of alpha-particle spectra," Nuclear Instruments and Methods in Physics Research Section A: Accelerators, Spectrometers, Detectors and Associated Equipment, vol. 498, no. 1-3, pp. 289-291, 2003.

[12] E. García-Toraño, "Current status of alpha-particle spectrometry," Applied Radiation and Isotopes, vol. 64, no. 10-11, pp. 1273-1280, 2006.

[13] F. Bayrakçeken, Z. Telatar, L. Tunçyürek, İ. Karaaslan, and A. Yaman, "High-resolution discrete absorption spectrum of $\alpha$-methallyl free radical in the vapor phase," Spectrochimica Acta Part A: Molecular and Biomolecular Spectroscopy, vol. 65, no. 1, pp. 143-146, 2006.

[14] S. Arı, M. C. Jiménez-Ramos, M. Villa, I. Vioque, G. Manjón, and R. García-Tenorio, "Numerical analysis of alpha spectra using two different codes," Applied Radiation and Isotopes, vol. 66, no. 6-7, pp. 808-812, 2008.

[15] M. P. R. Villa, C. J. G. Orellana, H. G. Velasco, and A. M. C. Lourtau, "Fast adaptive alpha-particle spectrum fitting algorithm based on genetically estimated initial parameters," Applied Radiation and Isotopes, vol. 60, no. 2-4, pp. 145-149, 2004.

[16] G. Velasco, "Uncertainty assessment in the analysis of alpha-particle spectra," Applied Radiation and Isotopes, vol. 49, no. 9-11, pp. 1241-1244, 1998.

[17] F. Vera Tomé, V. Gómez Escobar, and A. Martín Sánchez, "Study of the peak shape in alpha spectra measured by liquid scintillation," Nuclear Instruments and Methods in Physics Research Section A: Accelerators, Spectrometers, Detectors and Associated Equipment, vol. 485, no. 3, pp. 444-452, 2002.

[18] R. E. Abdel-Aal, “A programmable Gaussian random pulse generator for automated performance measurements, nuclear instruments and methods in physics research section A: accelerators, spectrometers," Detectors and Associated Equipment, vol. 276, no. 3, pp. 573-576, 1989.
[19] Z. Li, X.-G. Tuo, L. M. Zhe et al., "Statistical distribution-based detector response function of a Si (PIN) detector for $\mathrm{K} \alpha$ and K $\beta$ X-ray," Chinese Physics C, vol. 37, no. 1, Article ID 018202, 2013.

[20] D. Strul, R. B. Slates, M. Dahlbom, S. R. Cherry, and P. K. Marsden, "An improved analytical detector response function model for multilayer small-diameter PET scanners," Physics in Medicine and Biology, vol. 48, no. 8, pp. 979-994, 2003.

[21] B. T. David, H. W. L. Philip, L. Wayne, and D. V. John, Gaussian Random Number Generators, UCLA, Los Angeles, CA, USA, 1997.

[22] W. Dahlbom and J. Van Aarle, "PC-based high-precision nuclear spectrometry," Nuclear Instruments and Methods in Physics Research Section A: Accelerators, Spectrometers, Detectors and Associated Equipment, vol. 286, no. 3, pp. 439-442, 1990.

[23] Berkeley Nucleonic Corporation, PB-5 datasheet, vailable at: https://www.berkeleynucleonics.com/model-pb-5-precisionnim-pulse-generator.

[24] CAEN Digital Detector Emulator, web page, available at: https://www.caen.it/csite/CaenProd.

[25] A. Fernández Timón, M. Vargas, and A. Martin, “A method to reproduce alpha-particle spectra measured with semiconductor detectors," Applied Radiation and Isotopes, vol. 68, no. 4-5, pp. 941-945, 2010.

[26] E. Martín Sánchez, G. Bortels, P. Bauer et al., "A survey of the physical processes which determine the response function of silicon detectors to alpha particles," Nuclear Instruments and Methods in Physics Research Section A: Accelerators, Spectrometers, Detectors and Associated Equipment, vol. 339, no. 12, pp. 102-108, 1994.

[27] K. Bortels and R. Helmer, Gamma- and X-ray Spectrometry with Semiconductor Detectors, North-Holland, Amsterdam, Netherlands, 1988.

[28] J. C. Lozano, S. Madruga, and F. Fernández, "A function using cubic splines for the analysis of alpha-particle spectra from silicon detectors," Nuclear Instruments and Methods in Physics Research Section A: Accelerators, Spectrometers, Detectors and Associated Equipment, vol. 449, no. 1-2, pp. 356-365, 2000.

[29] Z. Fernández, X. Tuo, R. Shi, and J. Yang, “A statistical approach to fit Gaussian part of full-energy peaks from Si (PIN) and SDD X-ray spectrometers," Science China Technological Sciences, vol. 57, no. 1, pp. 19-24, 2014.

[30] C. M. Yang, C. C. Conti, and P. H. B. Becker, "Determination of HPGe detector response using MCNP5 for $20-150 \mathrm{keV}$ X-rays," Applied Radiation and Isotopes, vol. 64, no. 6, pp. 700-705, 2006.

[31] D. Ponikvar, "Generator of pseudo random pulses," Nuclear Instruments and Methods in Physics Research Section B: Beam Interactions with Materials and Atoms, vol. 83, no. 1-2, pp. 295-299, 1993.

[32] J. Gál, B. György, and J. Pálvölgyi, “A random tail pulse generator for simulation of nuclear radiation detector signals," Nuclear Instruments and Methods, vol. 171, no. 2, pp. 401-406, 1980.

[33] M. J. Koskelo, W. C. Burnett, and P. H. Cable, "An advanced analysis program for alpha-particle spectrometry," Radioactivity \& Radiochemistry, vol. 7, no. 1, pp. 23-36, 1996. 